\title{
LANGERHANS CELL HISTIOCYTOSIS: A CASE REPORT AND UPDATE ON TREATMENT MODALITIES
}

Hanapiah F, Windan TM, Saeed BMM, Rahman RA. Longerhans cell Histiocytosis: a case report and update on treatment modalities. Annal Dent Univ Malaya 1996; 3: 47-50

\begin{abstract}
Various treatment modalities exist for Langerhans cell histiocytosis $(\mathrm{LCH})$ due to its uncertain aetiology.. LCH patients are clinically divided into a single and multisystem group and each group warrants a different approach to treatment. However, histologically, CDla antigen and Birbeck's granules have been implicated in both types of the disease. This article therefore describes clinical presentations of different forms of the disease and the treatments contrived for them. The single system group is treated conservatively with either curettage of the localised lesion, intralesional steroid injection or in some cases radiotherapy. For the multisystem group, treatment consists af chemotherapeutic agents as either a single or combination therapy.
\end{abstract}

Key Words: Langerhans cell histiocytosis, Letterer-Siwe disease, Birbeck's granules.
Firdaus Hanapiah BDS (Otago)*

Tun Muzamal Wildan **

Bashir M.M. Saeed BDS (Khartoum)*

Rusdi Abdul Rahman BDS (Tanta)*

* Department of Oral \& Maxillofacial Surgery, Eastman Dental Institute, 256 Gray's Inn Road, London WC1 X8LD, United Kindom.

** 4th year Dental Student, Royal London Hospital, Dental Institute, Turner Street, London E1 2AD, United Kindom.

\section{INTRODUCTION}

Langerhans cell histiocytosis( $(\mathrm{CH})$ have been an enigma since its discovery by Hand in 1893 (1). It remained so for a very long time due to its wide clinical presentations, variable histopathological features and an uncertain aetiology. It was Lichtenstein in 1953 (2) who analyzed the range of clinical and pathological variations and concluded that the malady of diseases' were in fact a single entity and termed it "Histiocytosis X'. Histiocytosis for the proliferation of histiocyte-like cells while " $X$ " denotes the unknown aetiology. Nezelof in 1973 (3) was first to identify that these proliferative histiocytes were Langerhans cells. Subsequent developments in immunocytochemistry have positively proven this by the discovery that these histiocytes share a common surface antigen with normal Langerhans cells, the CDIa antigen (4). It is now accepted that Langerhans cell histiocytosis ( $\mathrm{LCH}$ ) is the most appropriate term to refer to the above disease.

\section{CLINICOPATHOLOGICAL PRESENTATIONS}

Little is known of the epidemiology of $\mathrm{LCH}$ primarily because it is a relatively rare disease. However Cartensen and Ornvold (5) found that the incidence rate of $\mathrm{LCH}$ is 1.08 in 200,000 in a survey involving a total population of 1 million children under the age of 15 in Denmark. The incidence of adult however is more difficult to fathom due to wider clinical presentations, often symptomless and under reporting by clinical specialists.

LCH is classified traditionally into 3 distinctive clinical presentations. The mildest of which is termed eosinophilic granuloma where symptoms of solitary or multiple bone lesions are usually reported. The second is the chronic disseminated form of LCH which is classically known as Hand-Schuller-Christian syndrome characterized by a triad of symptoms which includes intraosseous lesion, exophthalmos and diabetes insipidus. The third is the acute or subacute form of the disease which is known as Letterer-Siwe syndrome. It is characterized by rapid onset with widespread skeletal and extraskeletal lesions. It occurs usually in infants less than 3 years of age with severe clinical course and sometimes results in a fabl outcome. Not all cases however satisfy the above criteria. Lieberman (6) proposed a simpler classification in which LCH is classified as having either a unifocal or multifocal entity.

The histological picture is possibly the common denominator of the above diseases. Under light microscopy, the lesion is often described as an aggregation of histiocytic like cells arranged in a sheet like fashion superimposed with eosinophils in a fibrous connective tissue matrix. Under electron microscopy, Birbecks granules are characteristically present. Immunohistochemistry would show a positive labelling for CDla surface antigen.

\section{CASE REPORT}

A two year old Chinese boy was referred from Kuching General Hospital to both the Paediatric Department, Faculty of Medicine and the Oral and Maxillofacial Surgery Department, Faculty of Dentisty, University of Malaya, Kuala Lumpur, Malaysia in February 1992. The

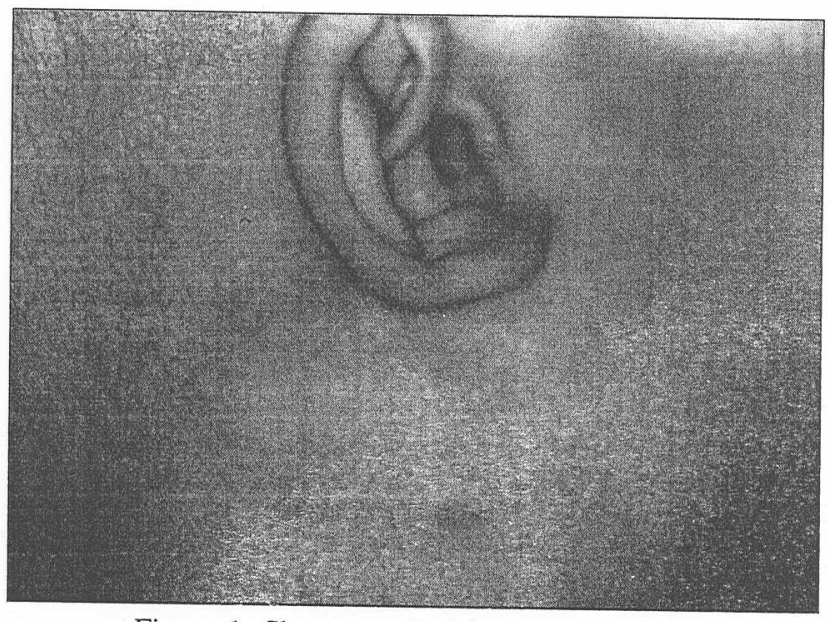

Figure 1: Shows cervical lymphadenopathy 


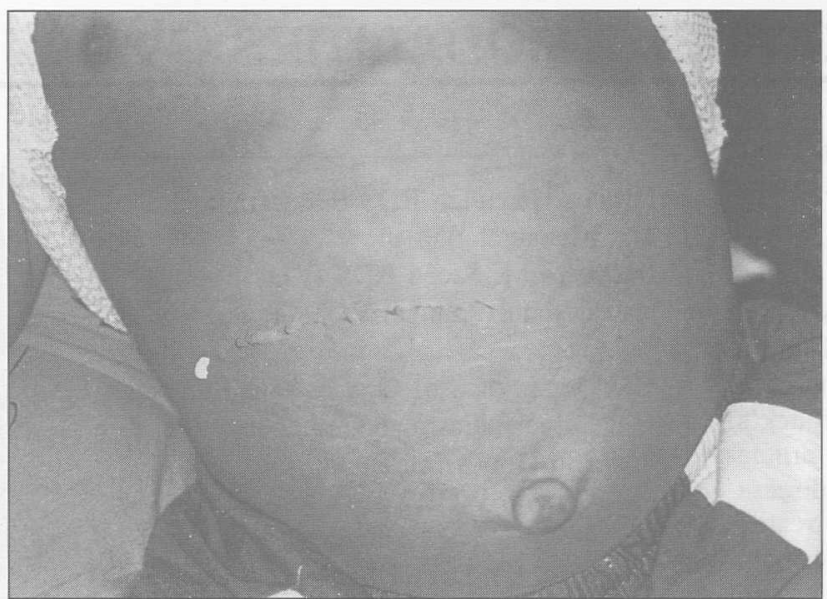

Figure 2: Shows a picture of the abdomen demonstrating hepatosplenomegaly

patient suffered recurrent bouts of fever and cervical lymphadenopathy. He was initially admitted to the Kuching General Hospital and treated with antibiotics but the bouts of fever persisted. He then went on to develop hepatosplenomegaly. At this point he was transferred to Kuala Lumpur for further assessment.

$\mathrm{He}$ is the youngest of nine siblings. His father is of Chinese origin while the mother is of Iban extract. No known medical problems of other members of the family were found. On examination we found that he had persistent and recurrent bouts of pyrexia, bilateral

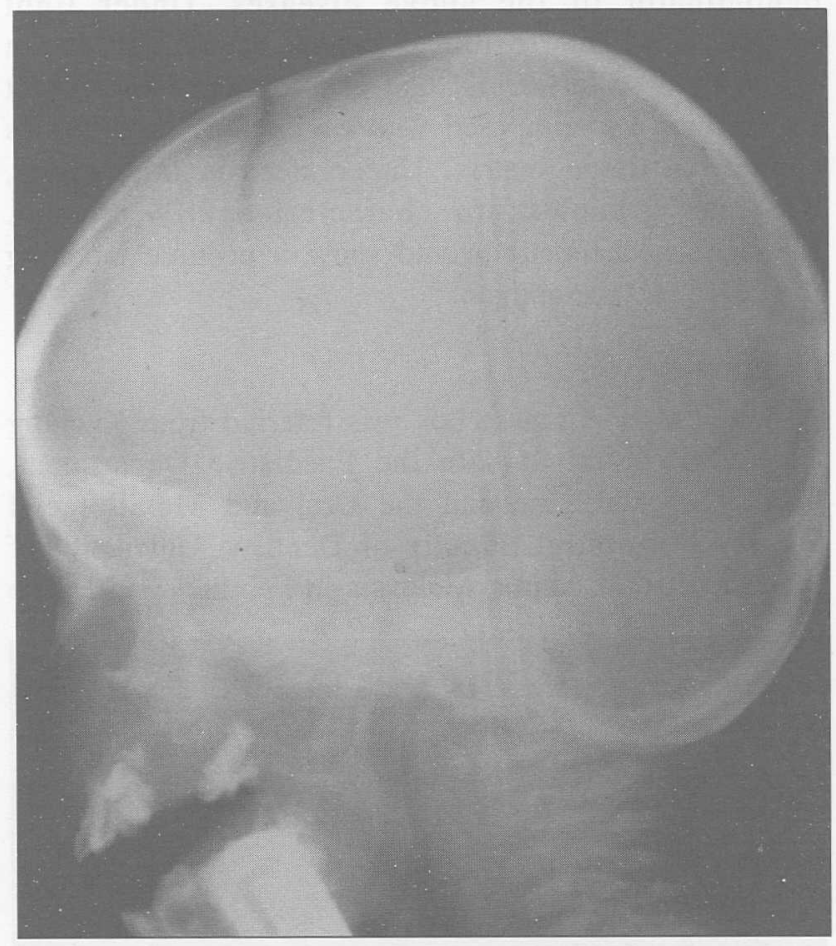

Figure 3: Lateral cephalogram demonstrating a distinct radiolucency of the maxilla and "floating in air" appearance of the upper teeth

cervical lymphadenopathy (Fig. 1), hepatosplenomegaly (Fig. 2) and seborrhoeic dermatitis of the scalp. Intraorally we found areas of granulomatous like lesion of the hard palate and the deciduous teeth were very mobile (Grades 2 and 3). Radiographic investigations were ordered - a Postero anterior(P.A.) skull radiograph and lateral cephalogram revealed a poorly defined radiolucency of the maxilla. The teeth showed characteristic "floating in air' appearance (Fig. 3). A full body skeletal survey, bone scan with TC 99 MDP showed "hot spots" localized to the maxillary bone. No other bone were found to be affected. A provisional diagnosis of Letterer-Siwe disease was considered most consistent to the above history and examination. However the occurrence of lymphoma was not ruled out. Biopsies were then taken from the palate and cervical lymph nodes which subsequently confirmed the diagnosis of LCH.

A full dearance of all deciduous teeth was done under general anaesthesia because it was decided that they could be a source of infection or the teeth may exfoliate and there is this risk of their being aspirated. The disease was treated with a single course of VP16 (Epipodophylotoxin Etoposide). Despite treatment, the patient died 4 months later from the disseminated form of the disease.

\section{DISCUSSION}

The treatment of $\mathrm{LCH}$ is somewhat a controversial issue. There are many variations amongst clinicians to their approach of treatment modalities. This is perhaps due to the uncertain aetiology of the disease. Recent developments have shown that $\mathrm{LCH}$ is a clonal disorder (7) suggesting that it may be neoplastic in origin. The variability of clinical manifestations from symptomless single system lesion to tnat of a life threatening multiple system disorder thus complicates the management even further.

Current approaches to the management of $\mathrm{LCH}$ is to divide the lesion into either a single or multiple system disease. A single system disease usually affect a single organ i.e. bone, lymph nodes. It is usually benign in nature and remission is likely. Therefore treatment for this disease has been more conservative. Curettage of the bone will usually lead to spontaneous remission.

Jones et al (8) and Ong et al (9) in their respective case reports have shown that intralesional infiltration of steroid ranging from 40 to $200 \mathrm{mg}$ methylprednisolone have been successful in the resolution of the isolated osteolytic lesion of the mandibular bone.

The use of radiotherapy have gradually gone out of favour amongst clinician possibly due to better understanding of the nature of the disease and the risk $(5 \%)$ of second tumour development (10). However, the use of low dose radiation is justifiable if $\mathrm{LCH}$ threatens critical organs such as lesions encroaching upon the optic nerve or the spinal cord where local infiltration of corticosteroid may not be possible.

In the multisystem disease, 2 major approaches exist - a conservative approach involving minimal therapy and. a more intensive chemotherapy plan. Based on observation by Broadbent (11), minimal therapy will lead to spontaneous recovery for chronic form of $\mathrm{LCH}$. However, 
evidence of fever, pain, immobility, failure to thrive and worsening condition of vital organs may indicate for a more aggressive approach (12). This may include the administration of methylprednisolone $(60 \mathrm{mg} / \mathrm{m} 2 /$ day $)$ given for a short period of time and followed by VP16, vincristine or vinblastine.

Another alternative approach stipulated by the German-ltalian prospective cooperative multicentre study group, the AIEOP-CNR-HX 83 (13), divides patients with multisystem disease into good and poor prognostic groups according to the absence or presence of organ dysfunction at the time of diagnosis. Treatment of good prognosis patients is to administer single agents sequentially to obtain favourable response. These are vinblastine $(6.5 \mathrm{mg} / \mathrm{m} 2$ i.v. weekly for three months) followed by doxorubicin $(20 \mathrm{mg} / \mathrm{m} 2 \mathrm{i}$.v. for 2 consecutive days every 3 weeks for 3 months) and finally VP16 (200 mg/m2 i.v. for 3 consecutive days, 3 weekly, at least for 3 months). For poor risk group, they will receive 9 courses of a 4 week treatment with a combination chemotherapy including vincristine, cydophosphamide, doxorubicin and prednisolone. This study concludes that response for single agents were effective in patient with good prognosis. In poor risk cases however, prognosis remain bad despite multi-agent therapy.

With greater understanding of the disease process researches have tried other approaches in the treatment of LCH. The Histiocyte Society have also established the Salvage Therapy Group to propose a study for the use of experimental drugs when the normal regimen have been proven ineffective (14). These approaches include the use of cyclosporin A (15) on the rationale that selective inhibition of the cellular immune response and cytokine mediated cellular activation would suppress the immunoarculatory effect of the disease. Alpha interferon have also been used in the hope of increasing NK cytotoxic activity. There is a case report of success in using this approach (16). However, no prospective clinical trial, as yet, have been initiated to show the efficacy of this form of treatment.

Bone marrow ablative therapy with bone marrow transplant (BMT) in the recurrent multisystem disease have been tried out in clinical practice A study (17) has reported some degree of success but the mortality rate due to either the recurrence of disease or the side effect of treatment is still unacceptably high.

Monoctonal antibody therapy of $\mathrm{LCH}$ patients exploits the potential role of monoclonal antibody directed against the CDla surface antigen of Langerhans cells. Kelly and Pritchard (18) were first to investigate the possibility of such treatment. The clinical application potential of this technique is immense either through a cytotoxic or immunoregulatory mode of treatment.

\section{CONCLUSION}

Langerhans cell histiocytosis have puzzled clinicians and scientists alike. Recent scientific breakthrough have shown that it is of neoplastic origin rather than a reactive disease. Clinical diagnosis may be difficult and often it is the dental surgeon who are first in line to notice this disease. LCH may mimic a wide range of clinical entities. It may present itself as juvenile or refractory periodontitis. It may be present as diabetes insipidus or a clinical condition similar to lymphoma. Therefore suspicious localized or generalized periodontal pocketing or bone loss must be investigated and the differential diagnosis of LCH must not be discounted.

Treatment of $\mathrm{LCH}$ is a contentious issue. However the consensus is to divide patients into single or multiple systems group. The single system group is treated more conservatively with either curettage of the localised lesion, intralesional steroid injection or in some cases radiotherapy, if the lesion threatens any vital organs. For the multisystem group, a more comprehensive approach is implemented primarily utilising VP16, vincristine or other chemotherapy agents as either a single or combination therapy. It must be pointed out that in situations where there is organ dysfunction, prognosis remain poor despite utilising all chemotherapy options. Novel therapeutics offer new potentials by opening new fronts in combatting LCH. This is only possible by further research in the quest for better understanding of this disease.

\section{Acknowledgement}

The authors wish to thank Sister Rathiyah Ahmad and her staff for their sincere efforts in assisting in the clinical work and the UMNO club of London for its facilities.

\section{REFERENCES}

1) Hand A. Polyuria and tuberculosis. Arch Paediatr 1893; 10: 673-5

2) Lichtenstein L. Histiocytosis $X$. Integration of eosinophilic granuloma of bone, "Letterer-Siwe Disease" and "Schuller-Christian Disease" as related manifestations of a single nosologic entity. A.M.A. Arch Pathol 1953; 56: 84-102

3) Nezelof C, Basset F, Rousseau MF. Histiocytosis X. Histiogenic arguments of a Langerhans cell origin. Biomed 1973; 18: 365-71

4) Chu T, Danglo GJ, Favara B, Ladisch S, Nesbit M, Pntchard J (Writing Group of Histiocyte Society). Histiocytosis syndrome in children. Lancet 1987; 1: 208-9.

5) Cartensten H, Omvold K. The epidemiology of Langernans cell histiocytosis in children in Denmark 1975-1989. Med Paediatr Oncol 1993; 21: 31-5 
6) Lieberman PH, Jones CR, Dargeon HWK, Begg CF. A reappraisal of eosinophilic granuloma of bone. Hand Schuller-Christian syndrome and Letterer-Siwe syndrome. Med 1969; 48: 375-400

7) Willman CL, McClain KL, Duncan MH, Kanapilly-Chavez L, Griffith BB, Issacson PG, Schnell B, Greswicks MVI, Favara BE. Molecular studies of clonality indicate that Langerhans cell histiocytosis is a clonal histiocytic neoplasm. Lab Invest 1993; 68: 128A

8) Jones LR, Toth BB, Cangir A. Treatment of solitary eosinophilic granuloma of the mandible by steroid injection: report of a case. J Oral Maxillofac Surg 1989; 47: 306-9

9) Ong ST, Lian CB,. Chronic disseminated Histiocytosis X: a case report. J Clin Paediatr Dent 1992; 17: 33-5

10) Greenberger JS, CrockerAC, Vawter G, Jaffe N, Cassady JR. Result of treatment of 127 patients with systemic histiocytosis (Letterer-Siwe syndrome, Schuiler-Christian syndrome and Multifocal Eosinophilc Granuloma). Medidne 1981; 60: 311-38

11) Broadbent V, Pritchard J. Histiocytosis X - current controversies. Arch Dis Child 1985; 60: 605-7

12) McLelland J, Broadbent V, Yeomans E, Malone M, Pritchard J. Langerhans cell histiocytosis: the case for conservative treatment. Med Ped Oncol 1990; 11: 99-105

13) Ceci A, De Terlizzi M, Coleila R, Loiacono G, Balucci D, Sarico G, Castello M, Testi AM, De Bernadi B, Indolfi P, Macchia P, Madon E, Mancini A, Rosati D. Langerhans cell hishocytosis in childhood: results from the Italian Cooperative AIEOP-CNR-HX 83 study. Med Pediatr Oncol 1993; 21: 259-64

14) Ladisch S, Gardner H. Treatment of Langerhans cell histiocytosis - evolution and current approaches. Brit J Cancer 1994; 70 (suppl XXIII): S41-S46

15) Mahmoud HH, Wang WC, Murphy SB. Cyclosporine therapy for advanced Langerhans cell histiocytosis. Blood 1991; 77: 721-5

16) Jakobson M, Kreuger A, Hagberg H, Sundstrom C. Treatment of Langerhans cell histiocytosis with alpha interferon. Lancet 1987; 1i: 208-9

17) Greinex HT, Storb R, Sanders JE, Petersen FB. Marrow transplantation for treatment of multisystem progressive Langerhans cell histiocytosis. Bone Marrow Transplant 1992; 10: 39-44

18) Kelly KM, Pritchard JP. Monocbnal antibody therapy in Langerhans cell histiocytosis - feasible and reasonable? Brit J Cancer 1994; 70 (suppl XXIII): S54-S55 